\title{
A Method for Assessing Customer Harmonic Emission Level Based on the Iterative Algorithm for Least Square Estimation*
}

\author{
Runrong Fan, Tianyuan Tan, Hui Chang, Xiaoning Tong, Yunpeng Gao \\ School of Electrical Engineering, Wuhan University, Wuhan, China \\ Email: rrfran0426@whu.edu.cn, tty@whu.edu.cn
}

Received June 2013

\begin{abstract}
With the power system harmonic pollution problems becoming more and more serious, how to distinguish the harmonic responsibility accurately and solve the grid harmonics simply and effectively has become the main development direction in harmonic control subjects. This paper, based on linear regression analysis of basic equation and improvement equation, deduced the least squares estimation (LSE) iterative algorithm and obtained the real-time estimates of regression coefficients, and then calculated the level of the harmonic impedance and emission estimates in real time. This paper used power system simulation software Matlab/Simulink as analysis tool and analyzed the user side of the harmonic amplitude and phase fluctuations PCC (point of common coupling) at the harmonic emission level, thus the research has a certain theoretical significance. The development of this algorithm combined with the instrument can be used in practical engineering.
\end{abstract}

Keywords: Harmonic Emission Levels; Harmonic Analysis; Least Square Estimation; Iterative Algorithm

\section{Introduction}

In the modern power grid system, the traditional power equipment has gradually been replaced by smart devices which were based on power electronics and other nonlinear element. Meanwhile, nonlinear loads of the user side were heavier and heavier. It made the harmonic pollution problems more serious and harmful to the safe operation of the power system. Besides, the users faced a great power loss. With the public's increasing emphasis on harmonic problems, the user's reasonable assessment of harmonic emission levels in public connection point became an important content of harmonic control [1].

The research of Emission level estimation of harmonic source was focused on harmonic source qualitative analysis and quantitative estimates at home and abroad: active power direction method [2] was widely used in engineering harmonic source qualitative analysis method. However, this method is affected deeply by the phase difference between the harmonic sources. And there is a big area of uncertainty, so it was not suitable for complex power systems. Harmonic source in quantitative analysis, also known as harmonic source emission level assess-

\footnotetext{
"Supported by "the Fundamental Research Funds for the Central Universities” (Grant No: 207-274592); Supported by NSFC (Grant No: 51007066)
}

ment, can be divided into intervention type and non-intervention type [3]. Intervention type need to inject into the system disturbance artificially, which not only increased the cost of harmonic analysis, also limited the scope of its application. Non-intervention type was different; it could use harmonic source fluctuations of itself to estimate the harmonic impedance in system without interfering with its normal operation. This method was simple to use, easy to the development of equipments. It has made a great difference in practice [4]. At present, the main non-invasive methods included fluctuations method $[5,6]$, the linear regression method $[8,9]$ and the reference impedance method [7]. Among them, fluctuations method and linear regression method were based on the condition that system harmonic impedance and equivalent systems invariant harmonic voltage source and the load harmonic impedance and equivalent load harmonic current source change greatly (or vice versa), it cannot be applied in a steady state harmonic source, and cannot applied in the condition while the system and user harmonic source volatility at the same [5-9]. The main disadvantage of the reference impedance method is that it needs to get more accurate prior reference impedance [7].

This paper proposed the iterative algorithm for least square estimation is based on the linear regression analysis of basic equation and the improvement equation. And 
we use this method to estimate harmonic emission level and obtain the real-time estimates of regression coefficients, and then calculate the level of the harmonic impedance and emission estimates in real time.

\section{The Principle and Basic Equation of Linear Regression Analysis Method}

The estimated value of the harmonic impedance and the customer emission level can be obtained by statistical analysis with linear regression analysis method, which can be divided into bilinear regression and 2 elements linear regression.

\subsection{Bilinear Regression Analysis Method}

The premise of bilinear regression method is that assuming the system operation is stable, harmonic impedance is purely inductive impedance; the resistance component is ignored, which means $I_{s}, X_{s}$ remains the same and $R_{s}$ is 0 . The principle diagram of the harmonic emission level at user side is shown in Figure 1.

The harmonic voltage equation at point PPC is:

$$
V_{s}=V_{p c c}+I_{p c c} Z_{s}
$$

Divide the vector into the real and imaginary parts, which are:

$$
\begin{aligned}
& V_{s x}=V_{p c c x}+I_{p c c x} Z_{s x}-I_{p c c y} Z_{s y} \\
& V_{s y}=V_{p c c y}+I_{p c c y} Z_{s x}+I_{p c c x} Z_{s y}
\end{aligned}
$$

The variation of harmonic currents at point PCC leads to the changes of harmonic voltage at user side, and the values of $V_{p c c x}, V_{p c c y}, I_{p c c x}, I_{p c c y}$ are obtained by measurement and analysis. Use linear regression analysis with these values to obtain the estimated value of $Z_{s y}$, $V_{s x}, V_{s y}$, and then equivalent harmonic voltage source $V_{s}$ and the harmonic impedance $Z_{s}$ can be got, so harmonic emission level of user side is:

$$
\begin{aligned}
V_{c_{-} p c c} & =V_{p c c}-\frac{V_{s} Z_{c}}{Z_{c}+Z_{s}} \\
& =V_{p c c}-\frac{V_{s}}{1+Z_{s} / Z_{c}} \\
& \approx V_{p c c}-V_{s}
\end{aligned}
$$

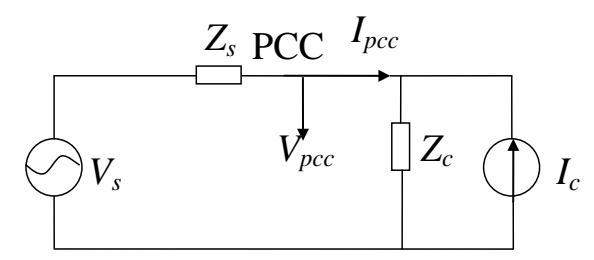

Figure 1. The principle diagram of the harmonic emission level at user side.

\subsection{Two Elements Linear Regression Analysis Method}

Bilinear regression ignores resistance component of harmonic impedance of system side, which may lead to the estimated results different from the actual harmonic impedance largely. In order to reduce the deviation of estimation value and the actual value, use the analysis methods with the least square method of type (2) and (3) to obtain the regression coefficient. The 2 elements linear regression model is as follows:

$$
\left\{\begin{array}{l}
y=b_{0}+b_{1} x_{1}+b_{2} x_{2}+\varepsilon \\
\varepsilon \sim N\left(0, \sigma^{2}\right)
\end{array}\right.
$$

\section{The Improvement of Linear Regression Analysis}

As showed in type (2) and (3), when the change of load harmonic source is only amplitude fluctuation, the real and imaginary parts of harmonic current at point PCC will synchronize increase or decrease. In order to reduce the correlation and improve the reliability of estimated value, the type (2) and (3) should transform, as follows:

$$
Z_{s y}=\frac{V_{p c c x}+I_{p c c x} Z_{s x}-V_{s x}}{I_{p c c y}}
$$

Put the type (6) into (3):

$$
\begin{aligned}
& I_{p c c x} V_{p c c x}+I_{p c c y} V_{p c c y} \\
& =-\left(I_{p c c x}^{2}+I_{p c c y}^{2}\right) Z_{s x}+I_{p c c x} V_{s x}+I_{p c c y} V_{s y}
\end{aligned}
$$

The type (3) is as follows:

$$
Z_{s x}=\frac{V_{s y}-I_{p c c x} Z_{s y}-V_{p c c y}}{I_{p c c y}}
$$

Put the type (8) into (2):

$$
\begin{aligned}
& I_{p c c y} V_{p c c x}-I_{p c c x} V_{p c c y} \\
= & \left(I_{p c c x}^{2}+I_{p c c y}^{2}\right) Z_{s y}+I_{p c c y} V_{s x}+\left(-I_{p c c x}\right) V_{s y}
\end{aligned}
$$

The matrix form of type (7) is:

$$
X=\left[\begin{array}{ccc}
-\left(I_{p c c x 1}^{2}+I_{p c c y 1}^{2}\right) & I_{p c c x 1} & I_{p c c y 1} \\
-\left(I_{p c c x 2}^{2}+I_{p c c y 2}^{2}\right) & I_{p c c x 2} & I_{p c c y 2} \\
\vdots & \vdots & \vdots \\
-\left(I_{p c c x n}^{2}+I_{p c c y n}^{2}\right) & I_{p c c x n} & I_{p c c y n}
\end{array}\right]
$$

The matrix form of type (3) is:

$$
X=\left[\begin{array}{ccc}
-\left(I_{p c c x 1}^{2}+I_{p c c y 1}^{2}\right) & I_{p c c y 1} & I_{p c c x 1} \\
-\left(I_{p c c x 2}^{2}+I_{p c c y 2}^{2}\right) & I_{p c c y 2} & I_{p c c x 2} \\
\vdots & \vdots & \vdots \\
-\left(I_{p c c x n}^{2}+I_{p c c y n}^{2}\right) & I_{p c c y n} & I_{p c c x n}
\end{array}\right]
$$


Use the transformed Equations (6) and (8) to solve the regression coefficients, in the inverse process; the correlation is reduced, so the reliability of results is improved. The above two equations are obtained after a series of data, through the matrix transformation and its inverse, and a complete algorithm for least squares. Completing algorithm in practical use needs large amount of memory and cannot be used in on-line identification.

\section{The Least Squares Estimation (LSE) Recursive Algorithm}

The basic idea of LSE recursive algorithm is that the new estimator is the sum of the last estimation and correction, the computation and storage of each step is small, offline or on-line identification can be allowed, and has the track time-varying parameters ability. When the $n$ measurement dates (samples) obtained, use the least squares complete algorithm to obtain the type $\hat{B}=\left(X^{T} X\right)^{-1} X^{T} Y$, where:

$$
\begin{gathered}
X_{n}=\left[\begin{array}{l}
\beta_{1}^{T} \\
\vdots \\
\beta_{n}^{T}
\end{array}\right] \\
Y_{n}=\left[\begin{array}{l}
y_{1} \\
\vdots \\
y_{n}
\end{array}\right]
\end{gathered}
$$

Make $P_{n}=\left(X_{n}^{T} X_{n}\right)^{-1}$, next moment, after a set of new data $\beta_{n+1}, y_{n+1}$ is obtained:

$$
\begin{aligned}
& P_{n+1}=\left(X_{n+1}^{T} X_{n+1}\right)^{-1}=\left[\left(X_{n}^{T}, \beta_{n+1}\right)\left(\begin{array}{c}
X_{n} \\
\beta_{n+1}^{T}
\end{array}\right)\right]^{-1}=\left(X_{n}^{T} X_{n}+\beta_{n+1} \beta_{n+1}^{T}\right)^{-1} \\
& X_{n+1}^{T} Y_{n+1}=\left(X_{n}^{T}, \beta_{n+1}\right)\left(\begin{array}{c}
Y_{n} \\
y_{n+1}
\end{array}\right)=X_{n}^{T} Y_{n}+\beta_{n+1} y_{n+1}
\end{aligned}
$$

Suppose $\mathrm{A}$ is $\mathrm{n}$-dimensional array, $\mathrm{B}$ and $\mathrm{C}$ are $n \times 1$ be got: dimensional vectors. $A, A+B C^{T}$ and $I_{m}+C^{T} A^{-1} B$ are full rank matrixes, and then:

$$
\left(A+B C^{T}\right)^{-1}=A^{-1}-A^{-1} B\left(I_{m}+C^{T} A^{-1} B\right)^{-1} C^{T} A^{-1}
$$

When $P_{n+1}$ meets the above conditions, the type can

$$
P_{n+1}=P_{n}-\frac{P_{n} \beta_{n+1} \beta_{n+1}^{T} P_{n}}{1+\beta_{n+1}^{T} P_{n} \beta_{n+1}}
$$

Thus, new estimates can be got:

$$
\begin{aligned}
\hat{B_{n+1}} & =\left(X_{n+1}^{T} X_{n+1}\right)^{-1} X_{n+1}^{T} Y_{n+1}=\left(P_{n}-\frac{P_{n} \beta_{n+1} \beta_{n+1}^{T} P_{n}}{1+\beta_{n+1}^{T} P_{n} \beta_{n+1}}\right)\left(X_{n}^{T} Y_{n}+\beta_{n+1} y_{n+1}\right) \\
& =P_{n} X_{n}^{T} Y_{n}-\frac{P_{n} \beta_{n+1} \beta_{n+1}^{T} P_{n}}{1+\beta_{n+1}^{T} P_{n} \beta_{n+1}} X_{n}^{T} Y_{n}+\left(P_{n}-\frac{P_{n} \beta_{n+1} \beta_{n+1}^{T} P_{n}}{1+\beta_{n+1}^{T} P_{n} \beta_{n+1}}\right) \beta_{n+1} y_{n+1} \\
& =B_{n}-\frac{P_{n} \beta_{n+1}}{1+\beta_{n+1}^{T} P_{n} \beta_{n+1}} \beta_{n+1}^{T} \hat{B}_{n}+\frac{P_{n} \beta_{n+1}}{1+\beta_{n+1}^{T} P_{n} \beta_{n+1}} y_{n+1}
\end{aligned}
$$

Above is the derivation process of recursive method. This paper adopts the following recursive formula:

$$
\left\{\begin{array}{l}
B(n+1)=B(n)+\gamma(n+1) P(n) X(n+1)\left[Y(n+1)-X^{T}(n+1) B(n)\right] \\
P(n+1)=P(n)-\gamma(n+1) P(n) X(n+1) X^{T}(n+1) P(n) \\
\gamma(n+1)=1 /\left[1+X^{T}(n+1) P(n) X(n+1)\right]
\end{array}\right.
$$

\section{Simulation Analysis}

This article established simulation model to meet the precondition of algorithm, in order to verify the linear regression analysis of three kinds of algorithms in the
MATLAB software (basic equation, modified equation, the iteration algorithm). It also estimated the harmonic impedance and harmonic emission level of the user. To facilitate the analysis, this article selects 5th harmonics as 
analysis object.

Harmonic impedance of system side is usually smaller than the user side, if impedance at the user side is unable to get accurate numerical, harmonic voltage formula emission levels of user side can be obtained by the above equation. Use the simulation of the modulus value level evaluation index for launch, namely $\left|V_{c_{-} p c c}\right| \approx\left|V_{p c c}-V_{s}\right|$.

In formula (19), $B(n)$ stand for the estimate value of last moment, $B(n+1)$ stand for new estimates of the current moment, the initial value of $B$ and $P$ select the first few data points to solve or set to $B(0)=0$ and $P(0)=\rho I$ effectively, $\rho$ takes a lot of positive scalar $\left(10^{5}-10^{8}\right), I$ stand for the Unit matrix, the influence of the initial value drops with the recursive number increasing, $\rho$ is set $10^{6}$ in the simulation.

\subsection{Basic Equation and the Modified Equation Algorithm Simulation}

The basic equation and the modified equation algorithm simulation model is shown in Figure 2, the system side harmonic source is set as constant 5th harmonic source, the user side set fluctuated 5th harmonic source, resistance, inductance are set as: $\mathrm{Rs}=5, \mathrm{Ls}=0.002 \mathrm{H}, \mathrm{Rc}=$ 15 , Lc $=0.02 \mathrm{H}$.

Set 5th harmonic amplitude of system side is 5A; the 5th harmonic amplitude of the user side (coefficient) varies with time as shown in Figure 3.

The real and imaginary parts of the 5th harmonic current and the real and imaginary parts of the 5th harmonic voltage are shown in Figure 4.

Use the simout module to store real and the imaginary part of 5th harmonic wave to work space, and then according to the basic Equations (2) and (3) and modified Equations (7) and (9) algorithm to write the procedures of the regression coefficient, the data shown in Tables 1 and 2.

a) When the user side only harmonic amplitude fluctuation, results were obtained as follows:

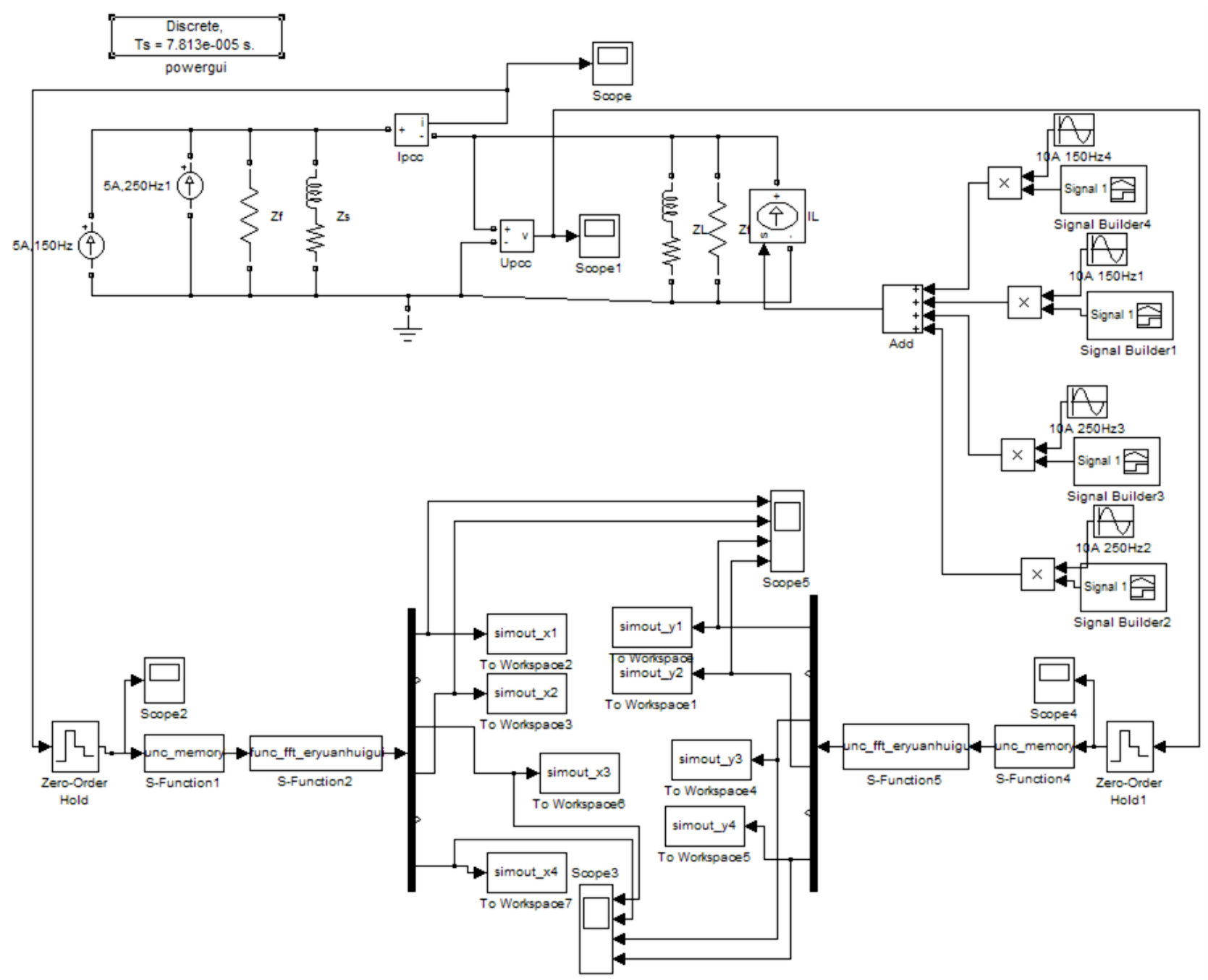

Figure 2. Linear regression analysis of complete simulation graph algorithm. 


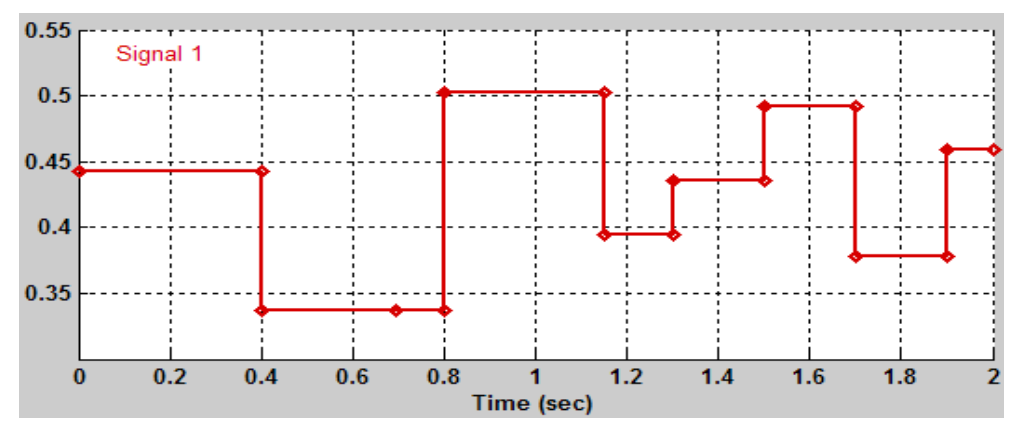

Figure 3. 5th harmonic amplitude variation curve of user side (coefficient).

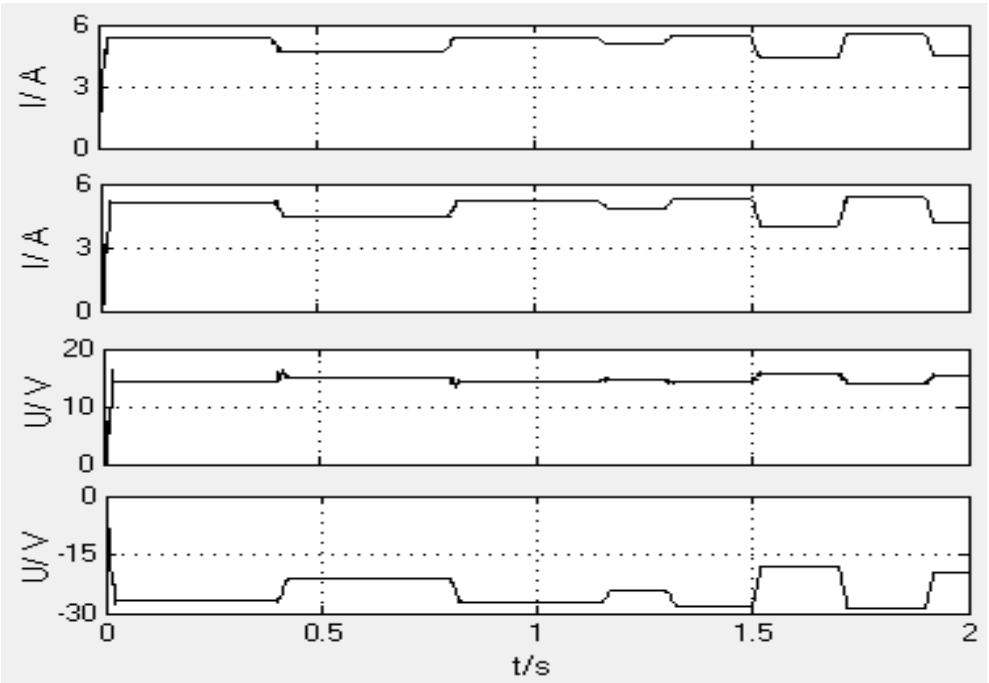

Figure 4. Real part and the imaginary part of 5th harmonic current and the real and imaginary part of 5th harmonic voltage.

Table 1. The user side only harmonic amplitude fluctuation data.

\begin{tabular}{cccccccc}
\hline Basic equation & Set value & Estimated value & Deviation (\%) & Modified equation & Set value & Estimated value & Deviation (\%) \\
\hline$V_{s x}(\mathrm{~V})$ & 25 & -3.798 & -115.19 & $Z_{s x}(\Omega)$ & 5 & 5.011 & 0.22 \\
$Z_{s x}(\Omega)$ & 5 & -26.541 & -630.82 & $V_{s x}(\mathrm{~V})$ & 25 & 24.957 & -0.172 \\
$Z_{s y}(\Omega)$ & 3.14 & -23.692 & -854.52 & $V_{s y}(\mathrm{~V})$ & 15.7 & 16.112 & 2.62 \\
$V_{s y}(\mathrm{~V})$ & 15.7 & 34.447 & 119.41 & $Z_{s y}(\Omega)$ & 3.14 & 3.203 & 2.01 \\
$Z_{s x}(\Omega)$ & 5 & -11.334 & -326.68 & $V_{s x}(\mathrm{~V})$ & 25 & 25.008 & 0.032 \\
$Z_{s y}(\Omega)$ & 3.14 & 22.416 & 613.89 & $V_{s y}(\mathrm{~V})$ & 15.7 & 16.102 & 2.56 \\
\hline
\end{tabular}

Table 2. Data when the user side of harmonic amplitude and phase fluctuations.

\begin{tabular}{cccccccc}
\hline Basic equation & Set value & Estimated value & Deviation (\%) & Modified equation & Set value & Estimated value & Deviation (\%) \\
\hline$V_{s x}(\mathrm{~V})$ & 25 & 24.999 & -0.004 & $Z_{s x}(\Omega)$ & 5 & 4.999 & -0.02 \\
$Z_{s x}(\Omega)$ & 5 & 5.0001 & 0.002 & $V_{s x}(\mathrm{~V})$ & 25 & 25.0001 & 0.0004 \\
$Z_{s y}(\Omega)$ & 3.14 & 3.221 & 2.58 & $V_{s y}(\mathrm{~V})$ & 15.7 & 16.104 & 2.57 \\
$V_{s y}(\mathrm{~V})$ & 15.7 & 16.104 & 2.57 & $Z_{s y}(\Omega)$ & 3.14 & 3.221 & 2.58 \\
$Z_{s x}(\Omega)$ & 5 & 5.0001 & 0.002 & $V_{s x}(\mathrm{~V})$ & 25 & 24.999 & -0.004 \\
$Z_{s y}(\Omega)$ & 3.14 & 3.220 & 2.55 & $V_{s y}(\mathrm{~V})$ & 15.7 & 16.105 & 2.58 \\
\hline
\end{tabular}


Improved regression coefficient equation algorithm to obtain estimates of the value of deviation is smaller, and put the average value (6) to emission level of the 5th harmonic wave the harmonic voltage at PCC (amplitude) is:

$\left|V_{c_{-} p c c}\right| \approx\left|V_{p c c}-V_{s}\right|$

$=|(24.957+25.008+\mathrm{j} 16.112+\mathrm{j} 16.102) / 2|=29.7247(\mathrm{~V})$

b) Harmonic amplitude and phase fluctuations in the user side

Estimation of the basic equation algorithm average calculation 5th harmonic wave, harmonic voltages in the PCC emission level (amplitude) is:

$$
\left|V_{c_{-} p c c}\right| \approx\left|V_{p c c}-V_{s}\right|=\mid(24.999+\mathrm{j} 16.104 \mid=29.737(\mathrm{~V}) ;
$$

Improved estimation equation algorithm average calculation 5th harmonic voltages in the PCC emission level (amplitude) is:

$$
\begin{aligned}
& \left|V_{c_{-} p c c}\right| \approx\left|V_{p c c}-V_{s}\right| \\
& =|(25.0001+24.999+\mathrm{j} 16.104+\mathrm{j} 16.105) / 2|=29.7377(\mathrm{~V})
\end{aligned}
$$

The data shows, when in user side harmonic amplitude fluctuates only and not phase fluctuations, regression coefficients obtained from the basic equation algorithm estimates a large deviation, the deviation is smaller by the estimation improved equation algorithm, namely improved equation algorithm can be used; when the amplitude and phase of the harmonic source user side fluctuate at the same time from the basic equations, and improved equation algorithm estimates the deviation is relatively small, the basic equations and the improved algorithm can be applied to equation.

\subsection{Least Squares Estimation Iterative Algorithm Simulation}

When LSE Iterative algorithm is applied (Set iteration coefficient matrix according to the improvement equation mentioned above), made user side harmonic source have only amplitude fluctuation, and the setting conditions of harmonic on both was the same to the algorithm that linear regression analysis complete disposable. Simulation Model is shown in Figure 5.

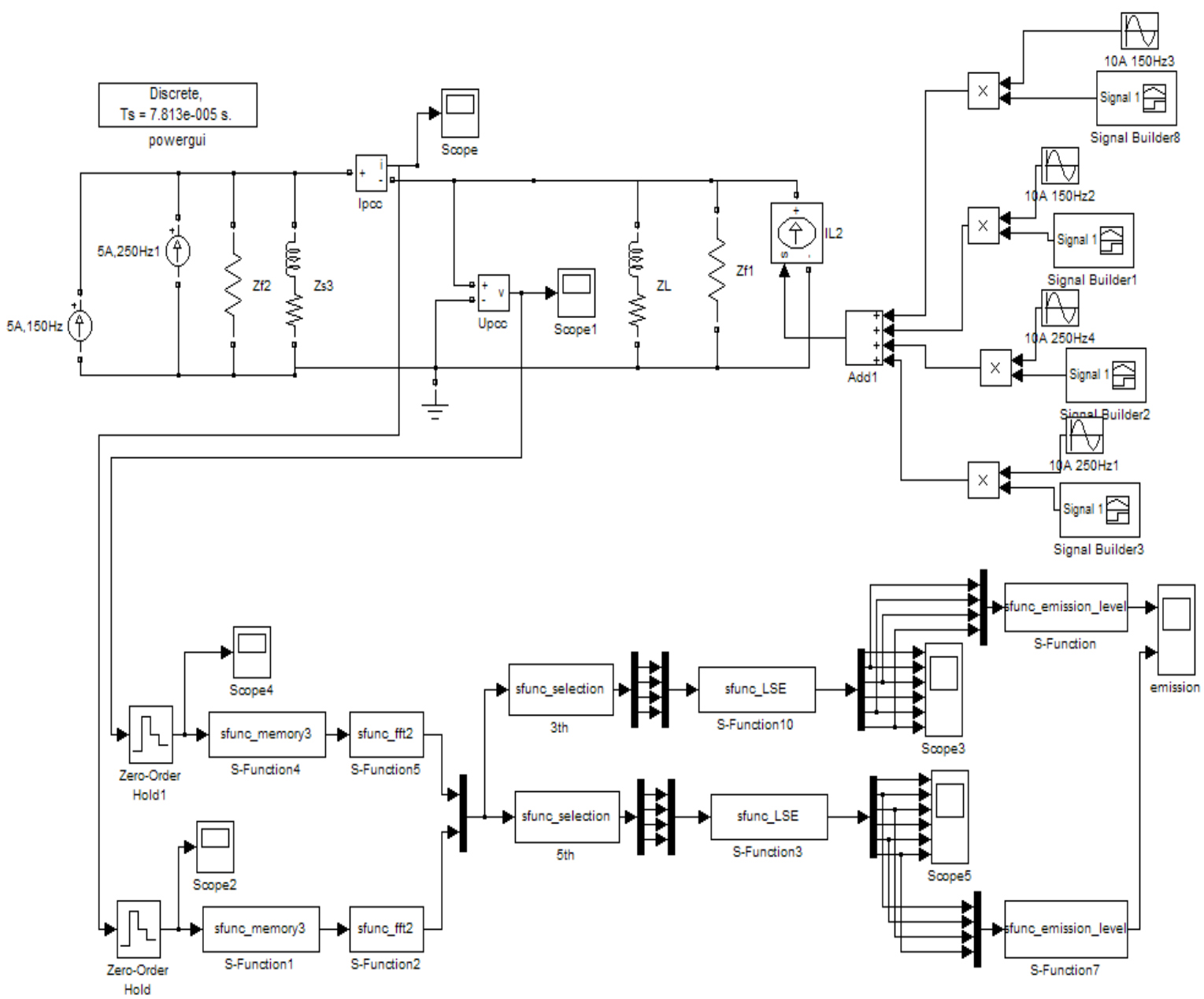

Figure 5. LSE iterative algorithm simulation model. 


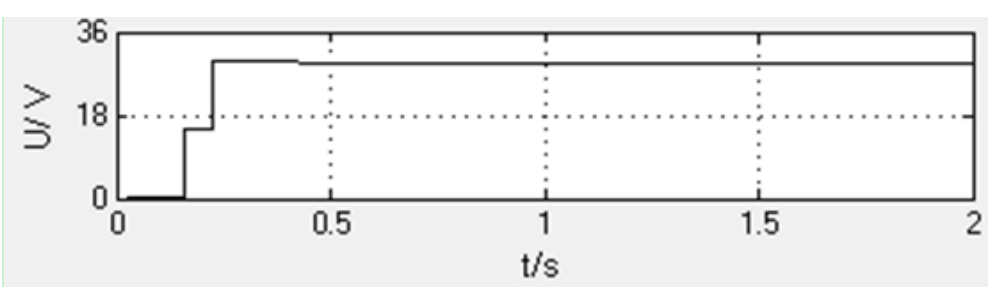

Figure 6. 5th harmonic emission level of user side at point of common coupling.

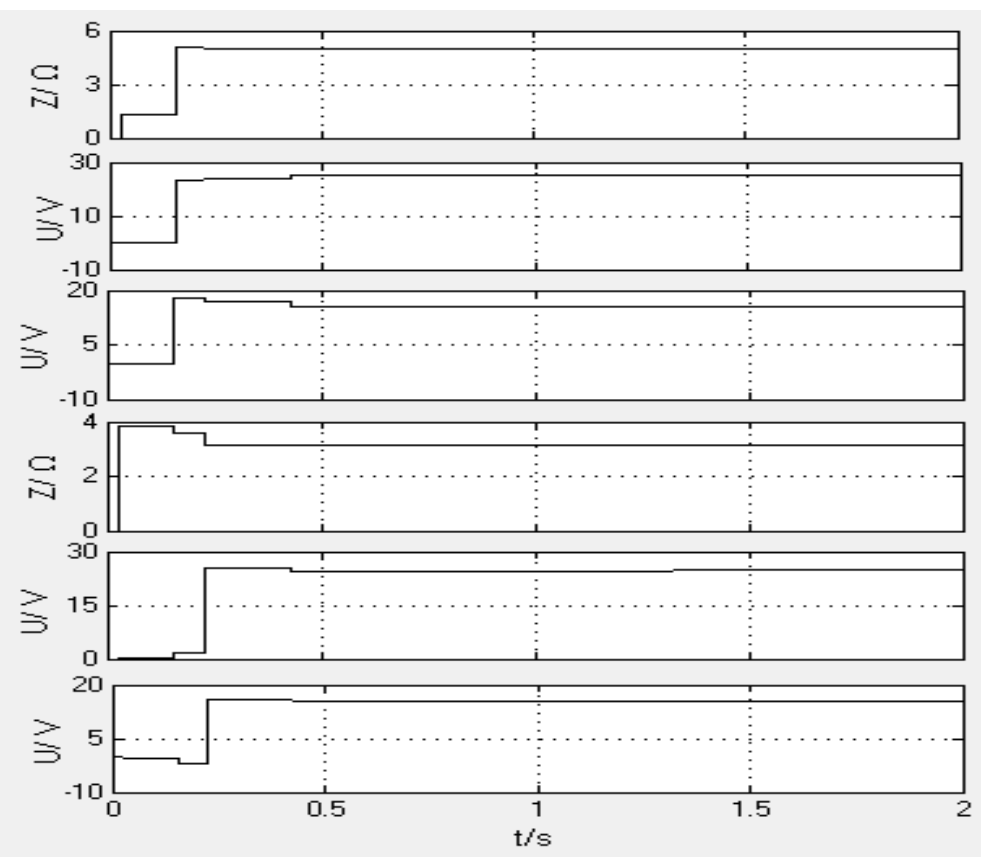

Figure 7. 5th harmonic regression coefficient estimates of recursive algorithm.

The 5th harmonic emission level (voltage amplitude) of user side at point of common coupling is shown in Figure 6.

The simulation results of LES recursive algorithm shown in Figure 7. Follow from top to bottom they are $Z_{s x}, V_{s x}, V_{s y}, Z_{s y}, V_{s x}, V_{s y}$.

We can see from the results of Iterative algorithm, the estimates of the third stationary of iterative algorithm became relatively stable, and its deviation is small compared with setting. Compared the two values (the estimate of the third stationary and the setting), we can get the Deviation range:

$$
\begin{aligned}
& Z_{s x}:-0.001 \%-0.001 \% ; \quad V_{s x}:-0.002 \%-0.008 \% ; \\
& V_{s y}: 2.91 \%-2.96 \% ; \quad Z_{s y}: 2.94 \%-2.97 \% ; \\
& V_{s x}:-0.006 \%-0.012 \% ; \quad V_{s y}: 2.93 \%-2.97 \%
\end{aligned}
$$

According to the data and figures above, we can conclude that, when the user side of the harmonic source fluctuation is small, iterative algorithm can get a small deviationist estimate and real-time, stable harmonic emission levels. What's more, with a small amount of data stored, running fast, online or offline running to get real-time harmonic estimates, iterative algorithm is ideal algorithm in the condition.

\section{Conclusion}

This paper derived iterative algorithm based on the basic equation and improved equation. This algorithm revised recognition result constantly on the advantage of new data; it got real-time harmonic impedance and harmonic emission level estimates. It calculated speed with small amount of data storage by this way. In addition, the iterative algorithm was without inversion process, so were the user side harmonic amplitude fluctuations and not only the phase fluctuations. There was no correlation between causing erroneous results and obtaining inverse problem. This algorithm will be improved and combined with the instrument development, it can be used in practical engineering, real-time monitoring of the PCC harmonic levels.

\section{REFERENCES}

[1] International Electric technical Commission (IEC), "Sub- 
Committee 77A, Electric magnetic Compatibility (EMC) Part 3 - 6: Limits Assessment of Emission Limits for the Connection of Distorting Installations to $\mathrm{MV}, \mathrm{HV}$ and EHV Power Systems,” British Standards Institution, United Kingdom, 2008.

[2] H. Yang, P. Porotte and A. Robert, "Assessing the Harmonic Emission Level from One Particular Customer," Proceedings of the 3rd International Conference on Power Quality, Vol. 2, No. 8, 1994, pp. 160-166.

[3] W. A. Omran, H. S. K. EI-Goharey, M. Kazerani and M. M. A. Salama, "Identification and Measurement of Harmonic Pollution for Radial and Nonradial Systems,” IEEE Transactions on Power Delivery, Vol. 24, No. 3, 2009, pp. 1642-1650.

http://dx.doi.org/10.1109/TPWRD.2009.2021043

[4] W. Xu, "Power Direction Method Cannot Be Used for Harmonic Source Detection,” IEEE Power Engineering Society Summer Meeting, 16-20 July 2000, Vol. 2.

[5] W. Xu and Y. L. Lin, "A Method for Determining Cus- tomer and Utility Harmonic Contribution at the Point of Common Coupling," IEEE Transactions on Power Delivery, Vol. 15, No. 2, 2000.

[6] C. Li, W. Xu and T. Tayjasanant, "A 'Critical Impedance' Based Method for Identifying Harmonic Sources,” IEEE Transactions on Power Delivery, Vol. 19, No. 2, 2004, pp. 671-678. http://dx.doi.org/10.1109/TPWRD.2004.825302

[7] Y. Xiao, J.-C. Maun, H. B. Mahmoud, T. Detroz and D. Stephane, "Harmonic Impedance Measurement Using Voltage and Current Increments from Disturbing Loads," 9th International Conference on Harmonics and Quality of Power, Vol. 1, Orlando, 1-4 Oct. 2000, pp. 220-225.

[8] W. Zhang and H.-G. Yang, "A Method for Assessing Harmonic Emission Level Based on Binary Linear Regression,” Proceedings of the CESS, Vol. 24, No. 6, 2004, pp. 50-53.

[9] Q. Che, H.-G, Yang, “Assessing the Harmonic Emission Level Based on Robust Regression Method,” Proceedings of the CSEE, Vol. 24, No. 4, 2004, pp. 39-42. 